\title{
Least Squares Differential Quadrature Method for the Generalized Bagley-Torvik Fractional Differential Equation
}

\author{
Constantin Bota (iD, Bogdan Căruntu (D), Mădălina Sofia Paşca, Dumitru Ţucu, \\ and Marioara Lăpădat \\ Department of Mathematics, Politehnica University Timisoara, Victoriei Square 2, Timisoara 300 006, Romania \\ Correspondence should be addressed to Bogdan Căruntu; bogdan.caruntu@upt.ro
}

Received 21 April 2020; Revised 12 June 2020; Accepted 17 June 2020; Published 16 July 2020

Guest Editor: Yi Qi

Copyright (c) 2020 Constantin Bota et al. This is an open access article distributed under the Creative Commons Attribution License, which permits unrestricted use, distribution, and reproduction in any medium, provided the original work is properly cited.

In this paper, the least squares differential quadrature method for computing approximate analytical solutions for the generalized Bagley-Torvik fractional differential equation is presented. This new method is introduced as a straightforward and accurate method, fact proved by the examples included, containing a comparison with previous results obtained by using other methods.

\section{Introduction}

Even though the fractional calculus is a relatively old concept, dating back from the time of the mathematicians L'Hospital and Leibniz in the $17^{\text {th }}$ century, it has seen a significant development in the last decades only. The fractional differential equations have numerous applications in biology, physics, chemistry, and engineering, which is why many scientists are concerned about finding more effective ways to solve them. Unfortunately, finding analytical solutions for fractional differential equations is often difficult or even impossible, which is why in recent years various numerical and approximate methods have been developed. One of the fractional differential equations with wide applicability in engineering is the Bagley-Torvik equation. This equation was introduced by Bagley and Torvik in [1] in 1984 to "model a motion of a rigid plate immersed in a Newtonian fluid" and in [2] for modeling the damping properties of the polymers. In engineering (construction, biotechnology, and chemistry), condensing polymers are widely used, and in their study, the Bagley-Torvik equation is the one employed $[3,4]$.

Bagley-Torvik-type fractional differential equations have been studied both numerically and analytically in numerous articles. Among the methods used to solve this equation, we mention the following: numerical methods [5], Adomian decomposition method [6], discrete spline methods [7], Haar wavelet method $[8,9]$, homotopy perturbation method [10], sinc collocation method [11, 12], cubic spline method [13], quadratic spline solution [14], B-spline collocation method [15, 16], Chebyshev collocation method [17], hybrid functions approximation [18], harmonic wavelets [19], predictor-corrector method of Adams type [20], spectral methods [21], fractional natural decomposition method [22], and finite element method [23].

The motivation of this paper is to introduce a new method for obtaining analytical approximate solutions for fractional differential equations. This method is obtained as a combination of the differential quadrature method (DQM) ([24]) and the least squares method (LSM), and we named it the least squares differential quadrature method (LSDQM). We applied this new method to find solutions of the following generalized Bagley-Torvik fractional differential equation [25]:

$$
A \cdot y^{\prime \prime}(x)+B(x) \cdot D_{x}^{\alpha} y(x)=f\left(x, y(x), D_{x}^{\beta} y(x), y^{\prime}(x)\right),
$$

where $y(x):[a, b] \longrightarrow \mathbb{R}, \alpha \in(1,2), \quad \beta \in(0,1)$, together with the conditions: 


$$
y(a)=\mu_{0}, \quad v_{1} \cdot y^{\prime}(a)+v_{2} \cdot y(b)=\mu_{1},
$$

where $\mu_{0}, \mu_{1}, \nu_{0}$, and $\nu_{1}$ are constants (for $\nu_{1}=0$, we obtain boundary conditions, while for $\nu_{2}=0$, we obtain initial conditions). $A \in \mathbb{R}, B(x)$ and $f(x)$ are given such that problems (1) and (2) satisfy the existence and uniqueness conditions for a continuous solution [25].

$D_{x}^{\gamma}$ denotes Caputo's fractional derivative:

$$
\begin{array}{r}
D_{x}^{\gamma} y(x)=\frac{1}{\Gamma(n-\gamma)} \cdot \int_{0}^{x}(x-\zeta)^{-(\gamma-n+1)} \cdot y^{(n)}(\zeta) d \zeta, \\
n-1<\gamma \leq n, n \in \mathbb{N}^{*} .
\end{array}
$$

In the next section, we will present the least squares differential quadrature method (LSDQM), and in the third section, some numerical examples are presented followed by the conclusions.

\section{The Least Squares Differential Quadrature Method}

First, we will consider a numerical mesh of the interval $I=$ $[a, b]$ by means of a partition $\Delta_{M}$ consisting of $M+1$ points: $a=x_{0}<x_{1}<x_{2}<\cdots<x_{M-1}<x_{M}=b p=0.007$. In order to find an approximate solution of the generalized Bagley-Torvik problems (1) and (2), we will compute the values of a certain functional (the functional (12) introduced in the following) at the point $x_{i}$.

To equation (1), we attach the following operator:

$$
\begin{aligned}
\mathscr{D}^{\alpha}(y(x))= & A \cdot y^{\prime \prime}(x)+B(x) \cdot D_{x}^{\alpha} y(x) \\
& -f\left(x, y(x), D_{x}^{\beta} y(x), y^{\prime}(x)\right) .
\end{aligned}
$$

We denote by $\tilde{y}(x)$ an approximate solution of equation (1). By replacing in $\mathscr{D}^{\alpha}$ the exact solution $y(x)$ with this approximate solution, we obtain the following reminder:

$$
\mathscr{R}(x, \tilde{y}(x))=\mathscr{D}^{\alpha}(\tilde{y}(x)) .
$$

Definition 1. We call an $\epsilon$-approximate solution of problems (1) and (2) related to the partition $\Delta_{M}$ an approximate polynomial solution, which satisfies the following relations:

$$
\begin{aligned}
\left|\mathscr{R}\left(x_{i}, \tilde{y}\left(x_{i}\right)\right)\right|<\varepsilon, \quad i=\overline{0, M} & \\
\tilde{y}(a) & =\mu_{0}, \quad v_{1} \cdot \tilde{y}^{\prime}(a)+v_{2} \cdot \tilde{y}(b)=\mu_{1} .
\end{aligned}
$$

Definition 2. We consider the sequence of polynomials:

$$
P_{N}(x)=\sum_{k=0}^{N} c_{k} x^{k}, \quad c_{k} \in \mathbb{R}, k=\overline{0, N}
$$

We call the sequence of polynomials $P_{N}(x)$ convergent to the solution of problems (1) and (2) if

$$
\lim _{N \longrightarrow \infty} \mathscr{D}^{\alpha}\left(P_{N}(x)\right)=0 \text {. }
$$

Taking into account the above definitions, we will compute $\epsilon$-approximate polynomial solutions for a Bagley-Torvik problem of the types (1) and (2) by taking the steps described in the following algorithm:

(i) Step 1. we will compute an approximate solution of the type

$$
T_{N}(x)=\sum_{k=0}^{N} \widetilde{c_{k}} x^{k}
$$

where the sequence of polynomials must also satisfy the boundary conditions:

$$
\begin{aligned}
T_{N}(a) & =\mu_{0}, \\
v_{1} \cdot T_{N}^{\prime}(a)+v_{2} \cdot T_{N}(b) & =\mu_{1},
\end{aligned}
$$

and the constants $\widetilde{c}_{k}$ are calculated in the following steps.

(ii) Step 2. From the boundary conditions, we obtain $\widetilde{c_{0}}$ and $\widetilde{c_{1}}$ as functions of $\widetilde{c_{2}}, \widetilde{c_{3}}, \ldots, \widetilde{c_{N}}$. Thus, the expression of $T_{N}(x)$ from now on will be a function of $x$ and $\widetilde{c}_{2}, \widetilde{c}_{3}, \ldots, \widetilde{c}_{N}$ only.

(iii) Step 3. We attach to problems (1) and (2) the functional $\mathscr{F}\left(\widetilde{c}_{2}, \widetilde{c}_{3}, \ldots, \widetilde{c}_{N}\right)$ :

$$
\mathscr{F}=\sum_{i=0}^{M} \mathscr{R}^{2}\left(x_{i}, T_{N}\left(x_{i}\right)\right)==\sum_{i=0}^{M}\left(A \cdot T_{N}^{\prime \prime}\left(x_{i}\right)+B(x) \cdot D_{x}^{\alpha} T_{N}\left(x_{i}\right)-f\left(x, T_{N}\left(x_{i}\right), D_{x}^{\beta} y(x), T_{N}^{\prime}\left(x_{i}\right)\right)\right)^{2} .
$$

(iv) Step 4. By minimizing the functional (12), we obtain the values of the coefficients $\widetilde{c}_{2}^{0}, \widetilde{c}_{3}^{0}, \ldots, \widetilde{c}_{N}^{0}$ which give the minimum of (12).

(v) Step 5. After computing $\widetilde{c}_{2}^{0}, \widetilde{c}_{3}^{0}, \ldots, \widetilde{c}_{N}^{0}$, we return to the boundary conditions and obtain the final value of $\tilde{c_{0}}$ and $\tilde{c_{1}}$.

(vi) Step 6. Finally, we replace the values $\widetilde{c}_{0}^{0}, \widetilde{c}_{1}^{0}, \widetilde{c}_{2}^{0}, \widetilde{c}_{3}^{0}, \ldots, \widetilde{c}_{N}^{0}$, calculate in the expression of
$T_{N}(x)$, and denote by $T_{N}^{0}(x)=\sum_{k=0}^{N} \widetilde{c}_{k}^{0} x^{k}$ the analytical approximate polynomial solution by LSDQM of problems (1) and (2).

The following convergence theorem is satisfied:

Theorem 1. The sequence of polynomials $T_{N}(x)$ satisfies the following relation: 


$$
\lim _{N \longrightarrow \infty} \mathscr{R}^{2}\left(x_{i}, T_{N}\left(x_{i}\right)\right)=0, \quad i=\overline{0, M} .
$$

Proof. Let $y(x)$ be an exact solution of problems (1) and (2), which means from hypothesis that there exists a sequence of polynomials $P_{N}(x)=\sum_{k=0}^{N} c_{k} x^{k}, \quad c_{k} \in \mathbb{R}, \quad k=\overline{0, N}$ converging to $y(x)$ (according to the Weierstrass theorem on Polynomial Approximation, see [24]), i.e., $\lim _{N \rightarrow \infty} P_{N}(x)=y(x), \quad \forall x \in I$. Taking into account Definition 2, this means that $P_{N}(x)$ satisfies

$$
\lim _{N \longrightarrow \infty} \mathscr{D}^{\alpha}\left(P_{N}(x)\right)=0 .
$$

Denoting by $c_{2}^{0}, c_{3}^{0}, \ldots, c_{N}^{0}$ the values of the coefficients $c_{k}$ which give the minimum of the functional (12), it follows that $\mathscr{F}\left(c_{2}^{0}, c_{3}^{0}, \ldots, c_{N}^{0}\right) \leq \mathscr{F}\left(c_{2}, c_{3}, \ldots, c_{N}\right)$. If $T_{N}^{0}(x)=$ $\sum_{k=0}^{N} \widetilde{c}_{k}^{0} x^{k}$ is the approximate solution by LSDQM (with $\widetilde{c}_{0}^{0}$ and $\widetilde{c}_{1}^{0}$ computed by using the initial conditions), we obtain

$$
\sum_{i=0}^{M} \mathscr{R}^{2}\left(x_{i}, T_{N}^{0}\left(x_{i}\right)\right) \leq \sum_{i=0}^{M} \mathscr{R}^{2}\left(x_{i}, P_{N}\left(x_{i}\right)\right) .
$$

Hence, $\quad \lim _{N \rightarrow \infty}\left(\sum_{i=0}^{M} \mathscr{R}^{2}\left(x_{i}, \quad T_{N}^{0}\left(x_{i}\right)\right)\right) \leq \leq \lim _{N \longrightarrow \infty}$ $\left(\sum_{i=0}^{M} \mathscr{R}^{2}\left(x_{i}, P_{N}\left(x_{i}\right)\right)\right)$.

We conclude that $\lim _{N \longrightarrow \infty} \mathscr{R}^{2}\left(x_{i}, T_{N}^{0}\left(x_{i}\right)\right)=0$.

\subsection{Error Estimation. We can rewrite equation (1) as}

$$
\begin{aligned}
& A \cdot y^{\prime \prime}(x)+B(x) \cdot D_{x}^{\alpha} y(x)-f_{1}\left(y(x), D_{x}^{\beta} y(x), y(x)\right) \\
& \quad=f_{2}(x) .
\end{aligned}
$$

We denote with $\mathscr{L}$ the following operator:

$$
\begin{aligned}
\mathscr{L}(y(x))= & A \cdot y^{\prime \prime}(x)+B(x) \cdot D_{x}^{\alpha} y(x) \\
& -f_{1}\left(y(x), D_{x}^{\beta} y(x), y^{\prime}(x)\right) .
\end{aligned}
$$

Thus, equation (1) becomes

$$
\mathscr{L}(y(x))=f_{2}(x) .
$$

Using (for simplicity) the notation: $\mathscr{R}(x)=$ $\mathscr{R}(x, \tilde{y}(x))=\mathscr{D}^{\alpha}(\widetilde{y}(x))$, in (4), we obtain

$$
\mathscr{R}(x)=\mathscr{L}(\tilde{y}(x))-f_{2}(x),
$$

with $\tilde{y}$ as an approximate solution for problems (1) and (2), which means as $\tilde{y}$ satisfies

$$
\begin{aligned}
\mathscr{L}(\tilde{y}(x))= & A \cdot y^{\prime \prime}(x)+B(x) \cdot D_{x}^{\alpha} y(x) \\
& -f_{1}\left(y(x), D_{x}^{\beta} y(x), y^{\prime}(x)\right)=f_{2}(x)+\mathscr{R}(x),
\end{aligned}
$$

together with the conditions:

$$
\tilde{y}(a)=\mu_{0}, \quad v_{1} \cdot \tilde{y}^{\prime}(a)+v_{2} \cdot \tilde{y}(b)=\mu_{1} .
$$

We define the error function in the following way: $\tilde{e}(x)=y(x)-\tilde{y}(x)$, where $y(x)$ is the exact solution for problems (1) and (2) and we obtain (using (18) and (19)) the differential equation for the error function:

$$
\mathscr{L}(\widetilde{e}(x))=\mathscr{L}(y(x))-\mathscr{L}(\widetilde{y}(x))=-\mathscr{R}(x),
$$

with the conditions:

$$
\tilde{e}(a)=0, \quad v_{1} \cdot \tilde{e}^{\prime}(a)+v_{2} \cdot \tilde{e}(b)=0 .
$$

The problem for the error function becomes

$$
\mathscr{L}(\widetilde{e}(x))=-\mathscr{R}(x) .
$$

Solving (24) together with (23) in the same manner as described above for problems (1) and (2), we obtain the approximation $\widetilde{e}(x)$. We will be able to determine the absolute maximum error:

$$
E=\max [|\widetilde{e}(x)|, \quad 0 \leq x \leq 1] .
$$

In this manner, we can estimate the error without knowing the exact solution of the initial problems (1) and (2).

Remark 1. We remark that the above theorem proves the convergence of LSDQM since as the degree of the polynomial increases the remainder corresponding to the approximation tends to zero. This fact is illustrated in the second example.

\section{Numerical Examples}

In this section, we will present some examples of problems including generalized Bagley-Torvik fractional differential equations together with boundary or initial conditions and problems solved using the least squares differential quadrature method (LSDQM).

3.1. Example 1. We consider the generalized Bagley-Torvik equation [26, 27]:

$$
\begin{aligned}
y^{\prime \prime}(x)+x^{(1 / 2)} \cdot D^{\alpha} y(x)+B y(x)= & -x^{(1 / 3)} \cdot y(x)-x^{(1 / 4)} \\
& \cdot D^{\beta} y(x)-x^{(1 / 5)} y(x) \\
& +f_{2}(x),
\end{aligned}
$$

together with the initial conditions: $y(0)=2, y^{\prime}(0)=0$, where $\quad f_{2}(x)=1-\left(x^{(1 / 2)} / \Gamma(3-\alpha)\right) \cdot x^{2-\alpha}-x^{(1 / 3)}$. $x-\left(x^{(1 / 4)} / \Gamma(3-\beta)\right) \cdot x^{2-\beta}+x^{(1 / 5)} \cdot\left(2-(1 / 2) \cdot x^{2}\right)$,

$\alpha=1.234$, and $\beta=0.333$. The exact solution of this problem is $y(x)=2-(1 / 2) \cdot x^{2}$.

Approximate solutions for this problem with absolute errors larger than $10^{-4}$ were proposed by El-Mesiry et al. in [26] and with absolute errors larger than $10^{-5}$ by $\mathrm{Li}$ and Zhao in [27].

We applied LSDQM to find an approximate analytical solution on the $[0,1]$ interval for (26).

In Step 1, we choose an approximate solution $\tilde{y}(x)$ of the following type:

$$
\tilde{y}(x)=\widetilde{c}_{0}+\widetilde{c}_{1} x+\widetilde{c}_{2} x^{2} .
$$


In Step 2, by using the initial conditions, we find $\widetilde{c}_{0}=2$ and $\widetilde{c}_{1}=0$, and the approximate solution becomes

$$
\tilde{y}(x)=2+\widetilde{c}_{2} x^{2}
$$

$$
\begin{aligned}
\mathscr{R}(\widetilde{y})= & \widetilde{c}_{2}\left(2 x^{(4 / 3)}+x^{(11 / 5)}+\frac{1000 x^{(633 / 500)}}{383 \Gamma(383 / 500)}+\frac{2000000 x^{(1917 / 1000)}}{1111889 \Gamma(667 / 1000)}+2\right)+x^{(4 / 3)} \\
& +\frac{x^{(11 / 5)}}{2}+\frac{x^{(633 / 500)}}{\Gamma(883 / 500)}+\frac{x^{(1917 / 1000)}}{\Gamma(2667 / 1000)}+1 .
\end{aligned}
$$

We consider the simplest possible partition $\Delta_{1}$ : $0=x_{0}<x_{1}=1$, and we attach to the equation the functional $\mathscr{F}\left(\widetilde{c}_{2}\right)=\sum_{i=0}^{1} \mathscr{R}^{2}\left(x_{i}, T_{1}\left(x_{i}\right)\right)$ (too long to be included here).

In Step 4, in order to find the minimum of this functional, we can compute the stationary points by equating to zero its first derivative (the computations are performed using the software Wolfram Mathematica). We obtain $\widetilde{c}_{2}=1 / 2$, and it is easy to show (by means of the second derivative) that this stationary point is indeed the minimum.

In the case of this problem, Step 5 is not needed since we already have the final values of $\widetilde{c}_{0}$ and $\widetilde{c}_{1}$.

Finally, in Step 6, we replace $\widetilde{c}_{0}=2, \widetilde{c}_{1}=0$, and $\widetilde{c}_{2}=1 / 2$ in the initial expression of $\tilde{y}(x)$, thus obtaining the exact solution of the equation: $\tilde{y}(x)=2-(1 / 2) \cdot x^{2}$.

We remark that, in the case of this example, we could choose any partition $\Delta_{M}$ since the particular form of the remainder (5) assures the fact that the stationary point is always $\widetilde{c}_{2}=1 / 2$ no matter how large $M$ is.

3.2. Example 2. We consider the fractional Bagley-Torvik boundary value problem [21]:

$$
\begin{aligned}
& y^{\prime \prime}(x)+D^{\alpha} y(x)+y(x)=f_{2}(x) \\
& f_{2}(x)=-\frac{4 c^{3} x_{1}^{3 / 2} F_{2}\left(1 ;(5 / 4),(7 / 4) ;-(1 / 4) c^{2} x^{2}\right)}{3 \sqrt{\pi}} \\
&-c^{2} \sin (c x)+\sin (c x) \\
& y(0)= 0 \\
& y(1)= \sin (c)
\end{aligned}
$$

where $[a, b]=[0,1], \alpha=1.5$, and $F$ is the generalized hypergeometric function.

The exact solution of this problem is $y(x)=\sin (c \cdot x)$.

We remark the fact that if the exact solutions of the problem is a polynomial function (as in the case of the first example), then, naturally, in applying the first step of our method, we have chosen an approximate polynomial solution (10) of the same degree as the exact solution. Since, in this example, the exact solution is not a polynomial, we computed approximate polynomial solutions of several degrees, and for each degree, we calculated the maximal value of the absolute error on the whole interval $[0,1]$ for $c=1$. The results presented in Table 1 together with a comparison with previous results from [21] clearly illustrate the convergence of the method.

The approximate polynomial solutions computed by LSDQM using an equidistant partition $\Delta_{100}$ are

$$
\begin{aligned}
\tilde{y}_{4}(x)= & 0.0196467 x^{4}-0.181975 x^{3}+0.00382023 x^{2}+0.999979 x \\
\tilde{y}_{5}(x)= & 0.00720937 x^{5}+0.00159936 x^{4}-0.167467 x^{3}+0.000129252 x^{2}+1 . x \\
\tilde{y}_{6}(x)= & -0.000658967 x^{6}+0.00918884 x^{5}-0.000526231 x^{4}-0.166518 x^{3}-0.0000146541 x^{2}+1 . x \\
\tilde{y}_{7}(x)= & -0.000172399 x^{7}-0.0000548963 x^{6}+0.00838255 x^{5}-0.0000217806 x^{4} \\
& -0.166662 x^{3}-3.209289 \cdot 10^{-7} x^{2}+1 . x \\
\tilde{y}_{8}(x)= & 0.0000117989 x^{8}-0.000219638 x^{7}+0.0000202955 x^{6}+0.00832241 x^{5} \\
& +3.233537 \cdot 10^{-6} x^{4}-0.166667 x^{3}+2.3498896 \cdot 10^{-8} x^{2}+1 . x
\end{aligned}
$$


TABLE 1: Maximal absolute errors for approximate polynomial solutions of various degrees obtained using LSDQM for example 2.

\begin{tabular}{lcr}
\hline Degree & Maximal error [21] & Maximal error LSDQM \\
\hline 4 & $3.4 \cdot 10^{-4}$ & $6.3979 \cdot 10^{-5}$ \\
5 & - & $1.3366 \cdot 10^{-6}$ \\
6 & - & $8.2879 \cdot 10^{-8}$ \\
7 & - & $1.3333 \cdot 10^{-9}$ \\
8 & $4.3 \cdot 10^{-7}$ & $7.3808 \cdot 10^{-11}$ \\
9 & - & $1.0573 \cdot 10^{-12}$ \\
10 & - & $6.5171 \cdot 10^{-14}$ \\
\hline
\end{tabular}

$$
\begin{aligned}
\tilde{y}_{9}(x)= & 2.40043968 \cdot 10^{-6} x^{9}+9.888419 \cdot 10^{-7} x^{8}-0.000199677 x^{7} \\
& +9.028126 \cdot 10^{-7} x^{6}+0.00833296 x^{5}+8.6069828 \cdot 10^{-8} x^{4}-0.166667 x^{3} \\
& +3.73299399 \cdot 10^{-10} x^{2}+1 . x \\
\tilde{y}_{10}(x)= & -1.3736474 \cdot 10^{-7} x^{10}+3.0878011 \cdot 10^{-6} x^{9}-4.5494814 \cdot 10^{-7} x^{8}-0.000198028 x^{7} \\
& -2.0494998 \cdot 10^{-7} x^{6}+0.0083334 x^{5}-1.31793399 \cdot 10^{-8} x^{4}-0.166667 x^{3}-4.5065671 \cdot 10^{-11} x^{2}+1 . x
\end{aligned}
$$

3.3. Example 3. Choosing in (1) $A=0, B(x)=0, f(x, y(x)$, $\left.D_{x}^{\beta} y(x), y^{\prime}(x)\right)=D_{x}^{\beta} y(x)+y^{2}(x)-1,[a, b]=[0,1], \mu_{0}=$ $\mu_{1}=\nu_{0}=\nu_{1}=0$, and $\alpha \in(0,1]$, we obtain the problem $[5,28]$ :

$$
\begin{aligned}
D_{x}^{\beta} y(x)+y^{2}(x)-1 & =0, \\
y(0) & =0 .
\end{aligned}
$$

This equation is actually a Riccati-type equation, and for $\alpha=1$, the exact solution of the problem is $y(x)=\left(e^{2 x}-1 / e^{2 x}+1\right)$.
Following the LSDQM steps presented in the previous section, we computed approximate polynomial solutions $\tilde{y}(x)$ of the 9th degree for problems (41) and (42) choosing in turn $\beta=1, \beta=0.9, \beta=0.8$, and $\beta=0.7$. The solutions are computed on the $[0,1]$ interval using again an equidistant partition $\Delta_{100}$. The results are presented in Figure 1 .

For example, the approximate polynomial solutions corresponding to $\beta=1$ is

$$
\begin{aligned}
\tilde{y}(x)= & -0.0106498 \cdot x^{9}+0.0567197 \cdot x^{8}-0.103159 \cdot x^{7}+0.0253074 \cdot x^{6}+ \\
& +0.125356 \cdot x^{5}+0.00150329 \cdot x^{4}-0.333491 \cdot x^{3}+7.88553637 \cdot 10^{-6} \cdot x^{2}+1 \cdot x
\end{aligned}
$$

The maximal absolute error corresponding to this approximation is $4.11 \cdot 10^{-9}$.

We remark that the results presented in Figure 1 are in good agreement with previously obtained results [5].

3.4. Example 4. While LSDQM, as most methods of this type, works best on small intervals, it can also be successfully employed in the case of large intervals. In order to illustrate this feature, we consider the Bagley-Torvik problem [6]:

$$
\begin{aligned}
y^{\prime \prime}(x)+D_{x}^{\alpha} y(x)+y(x) & =0, \\
y(0) & =1, \\
y^{\prime}(0) & =0,
\end{aligned}
$$

where $\alpha \in(1,2)$.

The exact solution of this problem is not known.

An approximate solution obtained by using the Adomian decomposition method was proposed in [6] by $\mathrm{V}$. Daftardar-Gejji and H. Jafari.

For $\alpha=1.5$, using an equidistant partition $\Delta_{10}$ on the interval $I=[0,20]$, we obtain the approximate analytical solution, presented in Figure 2:

$$
\begin{gathered}
\tilde{y}=-4.24381 \cdot 10^{-9} \cdot x^{9}+3.757992 \cdot 10^{-7} \cdot x^{8}-0.0000130166 \cdot x^{7}+0.00021244, \\
x^{6}-0.00133775 \cdot x^{5}-0.00503943 \cdot x^{4}+0.108157 \cdot x^{3}-0.404114 \cdot x^{2}+1 .
\end{gathered}
$$




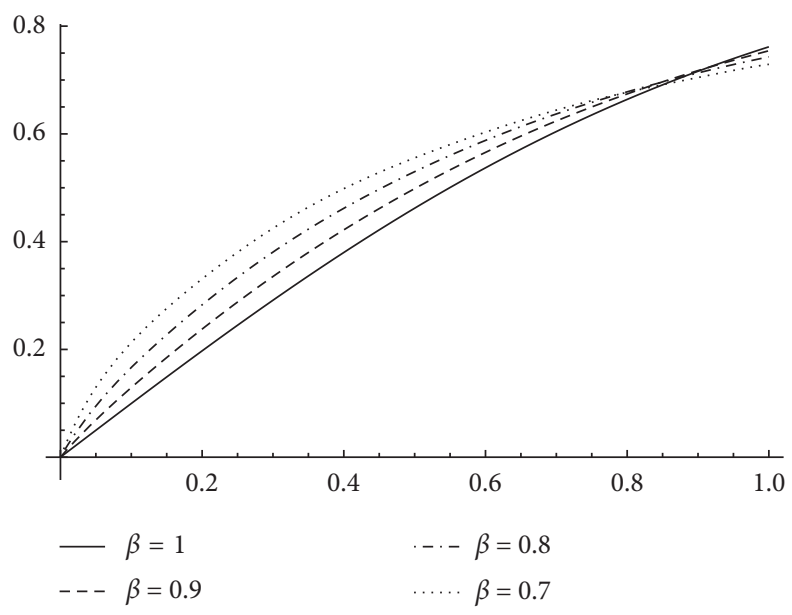

Figure 1: Approximate solutions for problems (41) and (42) corresponding to several values of $\beta$.

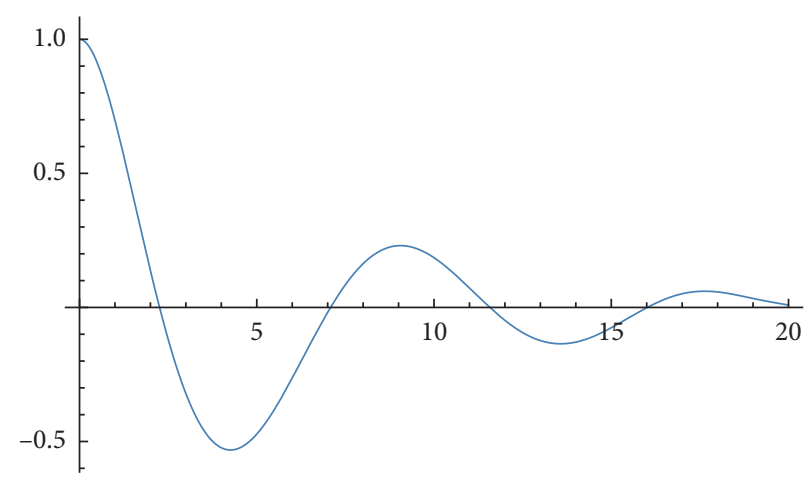

Figure 2: Approximate solution for the problems (44) and (45) corresponding to $\alpha=1.5$.

We remark that our result is in very good agreement with the one in [6].

\section{Conclusions}

In this paper, the application of the least squares differential quadrature method (LSDQM) to the Bagley-Torvik fractional differential equation is presented.

Due to the fact that the method is relative straightforward, the approximations may be obtained in a quick and simple manner. The numerical examples included clearly illustrate the accuracy of the method by means of comparisons with solutions previously computed by other methods. If the exact solution of the problem is itself a polynomial, then usually LSDQM is able to find the exact solution, as in the case of the first example. If not, in most of the cases, the solutions provided by LSDQM are not only more precise, but being of the simplest form (i.e., polynomial solutions), they are much easier to use in subsequent computation than many of the solutions provided by other methods.

\section{Data Availability}

All the necessary data are included in the paper.

\section{Conflicts of Interest}

The authors declare that there are no conflicts of interest.

\section{References}

[1] P. J. Torvik and R. L. Bagley, "On the appearance of the fractional derivative in the behavior of real materials," Journal of Applied Mechanics, vol. 51, no. 2, pp. 294-298, 1984.

[2] R. L. Bagley and P. J. Torvik, "Fractional calculus-a different approach to the analysis of viscoelastically damped structures," AIAA Journal, vol. 21, no. 5, pp. 741-748, 1983.

[3] T. Aleroev, S. Erokhin, and E. Kekharsaeva, "Modeling of deformation-strength characteristics of polymer concrete using fractional calculus," IOP Conf. Series: Materials Science and Engineering, vol. 365, Article ID 032004, 2018.

[4] B. H. E. Ibrahim, Q. Dong, and Z. Fan, "Existence for boundary value problems of two-term Caputo fractional differential equations," The Journal of Nonlinear Sciences and Applications, vol. 10, no. 2, pp. 511-520, 2017.

[5] H. Jafari, H. Tajadodi, H. Nazari, and C. M. Khalique, "Numerical solution of non-linear Riccati differential equations with fractional order," International Journal of Nonlinear Sciences and Numerical Simulation, vol. 11, pp. 179-182, 2010.

[6] V. Daftardar-Gejji and H. Jafari, "Adomian decomposition: a tool for solving a system of fractional differential equations," 
Journal of Mathematical Analysis and Applications, vol. 301, no. 2, pp. 508-518, 2005.

[7] W. K. Zahra and M. Van Daele, "Discrete spline methods for solving two point fractional bagley-torvik equation," Applied Mathematics and Computation, vol. 296, pp. 42-56, 2017.

[8] M. U. Rehman and R. A. Khan, "A numerical method for solving boundary value problems for fractional differential equations," Applied Mathematical Modeling, vol. 36, no. 3, pp. 894-907, 2012.

[9] S. Micula and C. Cattani, "On a numerical method based on wavelets for fredholm-hammerstein integral equations of the second kind," Mathematical Methods in the Applied Sciences, vol. 41, no. 18, pp. 9103-9115, 2018.

[10] Y.-G. Wang, H.-F. Song, and D. Li, "Solving two-point boundary value problems using combined homotopy perturbation method and green's function method," Applied Mathematics and Computation, vol. 212, no. 2, pp. 366-376, 2009.

[11] S. Alkan, K. Yildirim, and A. Secer, "An efficient algorithm for solving fractional differential equations with boundary conditions," Open Physics, vol. 14, no. 1, pp. 6-14, 2016.

[12] S. Yüzbasi, "Numerical solution of the Bagley-Torvik equation by the bessel collocation method," Mathematical Methods in the Applied Science, vol. 36, pp. 300-312, 2013.

[13] W. K. Zahra and S. M. Elkholy, "Cubic spline solution of fractional Bagley Torvik equation," Electronic Journal of Mathematical Analysis and Applications, vol. 1, no. 2, pp. 230-241, 2013.

[14] W. K. Zahra and S. M. Elkholy, "Quadratic spline solution for boundary value problem of fractional order," Numerical Algorithms, vol. 59, no. 3, pp. 373-391, 2012.

[15] K. Thula and P. Roul, "A high-order B-spline collocation method for solving nonlinear singular boundary value problems arising in engineering and applied science," Mediterranean Journal of Mathematics, vol. 15, 2018.

[16] H. Allouche, A. Tazdayte, and K. Tigma, "Highly accurate method for solving singular boundary-value problems via padé approximation and two-step quartic $B$-spline collocation," Mediterranean Journal of Mathematics, vol. 19, 2019.

[17] H. Jafari, A. Babaei, and S. Banihashemi, "A novel approach for solving an inverse reaction-diffusion-convection problem," Journal of Optimization Theory and Applications, vol. 183, no. 2, pp. 688-704, 2019.

[18] S. Mashayekhi and M. Razzaghi, "Numerical solution of the fractional bagley-torvik equation by using hybrid functions approximation," Mathematical Methods in the Applied Sciences, vol. 39, no. 3, pp. 353-365, 2016.

[19] C. Cattani, "A review on harmonic wavelets and their fractional extension," Journal of Advanced Engineering and Computation, vol. 2, no. 4, pp. 224-238, 2018.

[20] K. Diethelm and J. Ford, "Numerical solution of the bagleytorvik equation," BIT Numerical Mathematics, vol. 42, no. 3, pp. 490-507, 2002.

[21] E. H. Doha, A. H. Bhrawy, and S. S. Ezz-Eldien, "Efficient chebyshev spectral methods for solving multi-term fractional orders differential equations," Applied Mathematical Modelling, vol. 35, no. 12, pp. 5662-5672, 2011.

[22] M. S. Rawashdeh and H. Al-Jammal, "Numerical solutions for systems of nonlinear fractional ordinary differential equations using the FNDM," Mediterranean Journal of Mathematics, vol. 13, no. 6, pp. 4661-4677, 2016.

[23] M. Badr, A. Yazdani, and H. Jafari, "Stability of a finite volume element method for the time-fractional advection-diffusion equation," Numerical Methods for Partial Differential Equations, vol. 34, no. 5, pp. 1459-1471, 2018.

[24] C. Shu, Differential Quadrature and its Application in Engineering, Springer, New York, NY, USA, 2000.

[25] S. Stanek, "Two-point boundary value problems for the generalized bagley-torvik fractional differential equation," Central European Journal of Mathematics, vol. 11, no. 3, pp. 574-593, 2013.

[26] A. E. M. El-Mesiry, A. M. A. El-Sayed, and H. A. A. El-Saka, "Numerical methods for multi-term fractional (arbitrary) orders differential equations," Applied Mathematics and Computation, vol. 160, no. 3, pp. 683-699, 2005.

[27] Y. Li and W. Zhao, "Haar wavelet operational matrix of fractional order integration and its applications in solving the fractional order differential equations," Applied Mathematics and Computation, vol. 216, no. 8, pp. 2276-2285, 2010.

[28] B. Căruntu, C. Bota, M. S. Paşca, and M. Lăpădat, “Approximate solutions for Riccati differential equation of fractional order using the Least Squares differential quadrature method," Scientific Bulletin of the "Politehnica" University of Timisoara, vol. 64, no. 78, pp. 18-28, 2019. 